\title{
Medical image compression framework based on compressive sensing, DCT and DWT
}

\author{
Rahul Kher ${ }^{1 *}$, Yamini Patel ${ }^{2}$ \\ ${ }^{1}$ EC Department,G H Patel College of Engineering \& Technology, VallabhVidyanagar, Gujarat, India \\ ${ }^{2}$ PG Student, M. E. (Embedded Systems), G H Patel College of Engineering \& Technology,Vallabh Vidyanagar, Gujarat, India
}

\begin{abstract}
In this paper, CS based medical images compression framework has been presented. The discrete cosine transform (DCT) as well as discrete wavelet transform (DWT) have been used for finding the sparsity in the medical images. The comparison of the results obtained by combining DCT and DWT with CS for medical images- CT and MRI- has been presented.The CS \& DWT compression scheme achieved a maximum compression ratio (CR) of 3.01 with PSNR of 31.6732 dB and average CoC value of 0.9983 for an MRI image, whereas for CT image these values are 2.89, $31.7935 \mathrm{~dB}$ and 0.9987 , respectively. The CS and DCT based algorithm could obtain a maximum $\mathrm{CR}$ value of 1.5789 with average values (for both types of images) of PSNR, RMSE and CoC of $13.1774 \mathrm{~dB}, 56.0007$ and 0.8384 , respectively.
\end{abstract}

\section{Introduction}

Nowadays, compression is a necessary step of images storage and transmission and Medical images contain huge and vital information. It is necessary to compress medical images without losing the vital data. So, for that we are using discrete cosine transform, discrete wavelet transform. Normally the Shannon/ Nyquist sampling theorem states that when capturing the signal one must sample at least two times faster than signal bandwidth to avoid losing information. In fact, this principle underlies nearly all signal acquisition protocol used in consumer audio, visual electronics, medical imaging devices, and radio receivers and so on. However, it is common in such systems to use an anti-aliasing lowpass filter to band limit the signal before sampling.Compressive Sensing is a new method to capture and represent the compressible signal at the rate significantly below the Nyquist rate. Compressive sensing theory asserts that one can recover certain signals or images from far fewer samples or measurements than traditionally required [1].

The concept of compressed sensing was originally presented by David Donoho in 2006 [2] which states that in a digital signal or image, if $\mathrm{x}$ is an unknown vector and we need to measure $\mathrm{n}$ linear functions of $\mathrm{x}$ and reconstruct the same. If $\mathrm{x}$ has "sparse" representation, which could be decided by some orthonormal basis like wavelet, Fourier or DCT, then $\mathrm{x}$ can be reconstructed by $\mathrm{n}$ samples (for a signal) or pixels (for an image) as opposed to a routine $\mathrm{m}$ samples/ pixel, where $(\mathrm{n}<<\mathrm{m})$. The concept was further elaborated and appled by Candes and Wakin in 2008 by representing the compressed sensing as compressive sampling, acquiring the signal/ image with sampling rate which is below Nyquist rate.

In [3-10] authors have used the above mentioned, concept of compressive sensing for compressing the medical images like CT, MRI etc. using various transforms.In this paper, we implemented the medical images-CT and MRI- compression using DCT, DWT and CS.The compression performance is evaluated using parameters like peak signal to noise ratio (PSNR), root mean square error (RMS) and correlation coefficient $(\mathrm{CoC})$.

\section{Compressive sensing}

Using compressive sensing one can recover certain signals or images from far fewer samples than traditionally required [1]. Compressive sensing relies on two principles:

Sparsity- which pertains to the signals of interest, andIncoherencewhich pertains to the sensing modality

\section{Sparsity}

A signal is called sparse if most of its components are zero. Sparsity expresses the idea that the "information rate" of a continuous time signal may be much smaller than suggested by its bandwidth, or that a discrete-time signal depends on a number of degrees of freedom which is comparably much smaller than its (finite) length [1].

As observed, many real-world signals are compressible in the sense that they are well approximated by sparse signal after an appropriate change of basis. However, a very good example of naturally sparse picture is the one of the sky during night, which has all pixels black except a few white dots (starts). Real world images are not usually sparse but can be translated into suitable domain to achieve sparsity. This explains why compression techniques such as JPEG, MPEG, or MP3 work so well in practice. For example, JPEG relies on the sparsity of images in the discrete cosine basis or wavelet basis and achieves compression by only storing the largest discrete cosine or wavelet coefficients.

Correspondence to: Rahul Kher, EC Department,G H Patel College of Engineering \& Technology, Vallabh Vidyanagar, Gujarat, India; E-mail: rahul2777@gmail.com

Key words: Compressive sensing; discrete wavelet transform (DWT); discrete cosine transform (DCT); PSNR; RMS; CoC

Received: January 15, 2017; Accepted: February 16, 2017; Published: February 18,2017 
For representing a signal in a sparse form, we would like to project it onto a convenient basis. The signal of interest $x$, having a sparse .....] such that $\mathrm{x}=\Psi$ s which can be mathematically expressed as

$$
x=\sum_{i=1}^{N} s_{i} \Psi_{i}
$$

Where " $s$ " is the coefficient vector for " $x$ " under the transformation basis $\Psi$.

\section{Incoherence}

This property demands that the measurement and sparsifying basis are incoherent i.e. the signal which has a sparse representation in a sparsifying basis must spread out in the measurement basis [1]. Within the Compressive sensing framework, low coherence between and translates to fewer samples required for reconstruction of signal. An example of low coherence measurement/representation basis pair is sinusoids and spikes that are incoherent in any dimension, and can be used for compressively sensing Signals having sparse representation in terms of sinusoids.

If our measurement matrix $\Psi$ were a square matrix, then we may have employed an identity matrix for the sake of sampling the signals. Clearly, this is the case of our traditional uniform sampling. But there is no way to adjust an identity matrix for our measurement matrix since it is a short, fat matrix i.e. number of measurements $M<<$ Signal length N.

Also, there must be least correlation between the elements of the sensing and the dictionary matrices as suggested by the incoherence property in section 2.3.2. The lesser the coherence between $\Psi$ and $\Phi$, fewer the number of measurements needed to recover the signal.

Mathematically, the coherence between the sensing basis $\Phi$ and the representation basis $\Psi$ is given by [1]

$$
\mu(\Phi, \Psi)=\sqrt{n} \cdot \max \left|\Phi_{k} \Psi_{k}\right|
$$

It follows from linear algebra that,

$$
1 \leq \mu(\Phi, \Psi) \leq \sqrt{n}
$$

Here, $\mu$ indicates the correlation between the sensing matrix and the representation matrix. Lesser the value of coefficient of correlation, better the results in the sense that we need the acquisition of very lesser number of samples i.e. general linear measurements.

An example of low coherence measurement basis pair is sinusoids and spikes that are incoherent in any dimension, and can be used for compressively sensing Signals having sparse representation in terms of sinusoids [1]. Other common pairs are indicated in Table 1.

\section{Recovery}

Generally, L0, L1, L2 minimization methods are used for reconstruction. But if signal is sufficiently sparse then $\mathrm{L} 1$ minimization provides best solution [1].

We take 11-norm i.e. sum of absolute values of coefficients. We don't pick a solution that is sparsest, or that has least energy. But we pick the one with smallest sum of magnitudes. Reason for doing this is that 11 -norm is convex and hence, it becomes a linear programming problem and we get a lot of standard linear programming tools to solve it unlike 10-norm. Moreover, quite surprisingly, 11-norm gives almost the same result as the 10 -norm [9].

Thus, the 11-norm essentially convexifies the 10-norm and is given as
Table 1. Selection of Low coherence pairs

\begin{tabular}{|c|c|}
\hline $\boldsymbol{\Phi}$ & $\boldsymbol{\Psi}$ \\
\hline Spike Basis & Fourier Basis \\
\hline Spike Basis & Sinusoids \\
\hline Noiselet & Wavelets \\
\hline Gaussian or Binary_1 & Any fixed \\
\hline
\end{tabular}

$$
\min || x||_{1}=\min \sum_{i=1}^{N}\left|x_{i}\right|
$$

Previous we discuss that Medical images carry huge and vital information. It is necessary to compress the medical images without losing its vital-ness. Wavelet transform is preferable in the field of compression because of much more information of image can be retraced by it than any other transform [3]. There are many different types of wavelets have been used for compression and we have done quality analysis on reconstructed image.

Wavelet transform is a mathematical tool used to represent images in a domain in which it may be manipulated more effectively or in another word the main purpose of wavelet transform is to change the data from time-space domain to time-frequency domain which makes batter compression result.

The fundamental idea behind wavelets is to analyze the signal or image at different scales or resolutions, which is called multiresolution. Wavelets are a class of functions used to localize a given signal in both space and scaling domains. A family of wavelets can be constructed from a mother wavelet. Compared to Windowed Fourier analysis, a mother wavelet is stretched or compressed to change the size of the window. In this way, big wavelets give an approximate image of the signal, while smaller and smaller wavelets zoom in on details. Therefore, wavelets automatically adapt to both the high-frequency and the lowfrequency components of a signal by different sizes of windows. Any small change in the wavelet representation produces a correspondingly small change in the original signal, which means local mistakes will not influence the entire transform.

Wavelet Transforms are based on small waves, called Wavelets, of varying frequency and limited duration. Wavelet analysis can be used to divide the information of an image into approximation and detail sub signals. The approximation sub signal shows the general trend of pixel values, and three detail sub signals show the vertical, horizontal and diagonal details or changes in the image. If these details are very small, then they can be set to zero without significantly changing the image [6].

As a result, there are 4 sub-band $(A, H, V, D)$ images at each scale. The sub-band A is used for the next 2D DWT. The first, second and third level decomposition of image with the help of wavelet transform, here is showing first level of decomposition with four different frequency levels.

Now taken decomposition of A1 and achieved second level of decomposition and further got seven different frequency levels(Table 2).

And another time taken decomposition of A2 and achieved third level of decomposition and further got different frequency levels(Table 3).

\section{Discrete cosine transform}

A discrete cosine transform (DCT) expresses a finite sequence of data points in terms of a sum of cosine functionsoscillating at 
Table 2. First level decomposition

\begin{tabular}{|l|l|}
\hline A1 & H1 \\
\hline V1 & D1 \\
\hline
\end{tabular}

Table 3. Second level decomposition

\begin{tabular}{|c|c|c|}
\hline A2 & H2 & H1 \\
\hline V2 & D2 & D1 \\
\hline
\end{tabular}

different frequencies. The DCT is like the discrete Fourier transform: it transforms a signal or image from the spatial domain to the frequency domain.

DCT is a transform coding method comprising four steps. The source image is first partitioned into sub-blocks of size $8 \times 8$ pixels in dimension. Then each block is transformed from spatial domain to frequency domain using a 2-D DCT basis function. The resulting frequency coefficients are quantized and finally output to a lossless entropy coder. DCT is an efficient image compression method since it can decorrelate pixels in the image.

Here is the equation for $1-$ DCT

$$
x_{k}=\frac{1}{2}\left(x_{0}+(-1)^{k} x_{N-1}\right)+\sum_{n=1}^{N-2} x_{n} \cos \left[\frac{\pi}{N-1} n_{k}\right]
$$

Where $\mathrm{k}=0,1 \ldots \ldots . \mathrm{N}-1$

Following is the equation for 2-DCT:

$$
x_{k}=\sum_{n=0}^{N-1} x_{n} \cos \left[\frac{\pi}{N}\left(n+\frac{1}{2}\right) k\right]
$$

Here is the Figure1 which gives the basic idea of DCT

\section{Experimental results}

In this section, the experimental (simulation) results for medical imagecompression using CS and DWT and CS and DCT has been presented. These algorithms are applied on a sample MRI (image 1) and CT (image 2) images, one each, and their performance is evaluated using PSNR, RMSE and CoC.All the simulations are performed using MATLAB software run on a core i7 CPU.

\section{A. CS and DWT based Results (Figure 2, 3 and Table 5)}

\section{B. CS and DCT based Results}

Now the second method is use compressive sensing in discrete cosine transform. In this method, first we apply the discrete cosine transform and get the sparsity image then use this image in compressive sensing algorithm. In this algorithm first the set of sparse components are multiplied with the random Gaussian matrix. Here the size of Gaussian matrix is taken as $\mathrm{MX} \mathrm{N}$, where the $\mathrm{M}$ is taken as per the required percentage of measurement which is first generated randomly and finally converted to the orthogonal matrix. This matrix, multiplied with the sparse components, provides the set of compressed data of the image using compressed sensing. Now to reconstruct the set of data L1 minimization is used and reconstruct the image (Figures 4, 5 and Table 6).

\section{Conclusion}

In this paper, a medical image compression framework using compressive sensing, discrete wavelet transform and discrete cosine transform has been presented. A sample CT and MRI image has

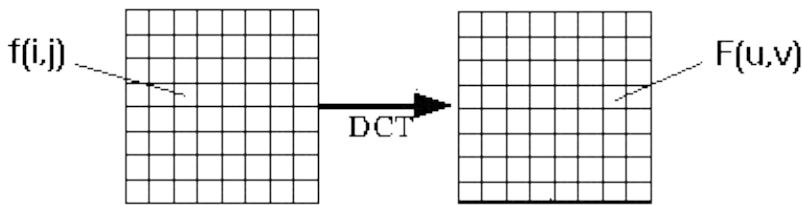

Figure 1. Discrete cosine transform

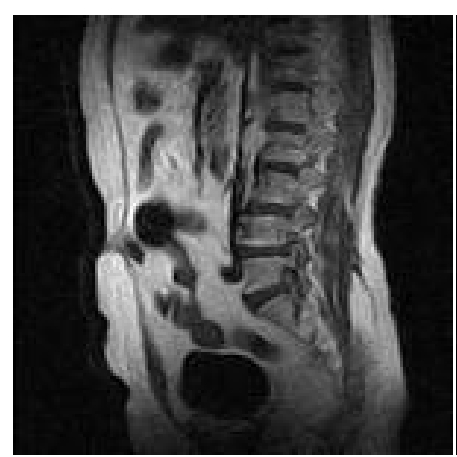

(a)

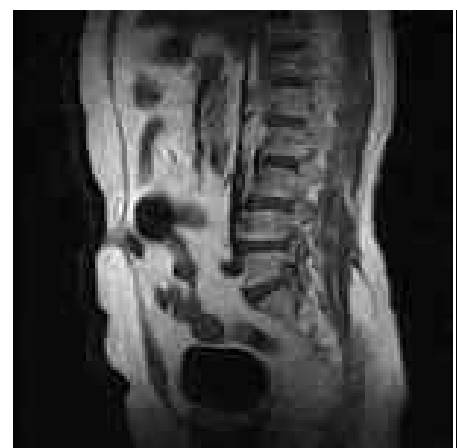

(c)

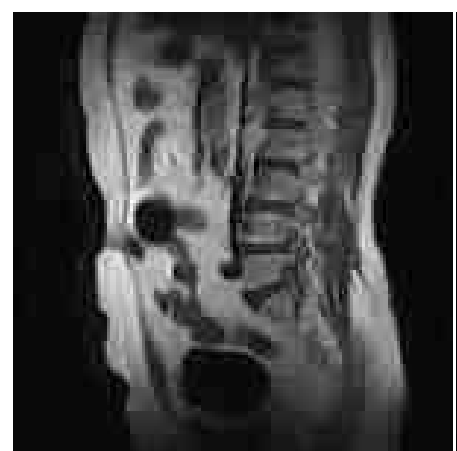

(e)

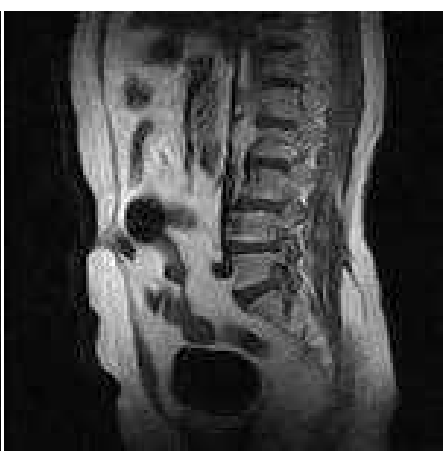

(b)

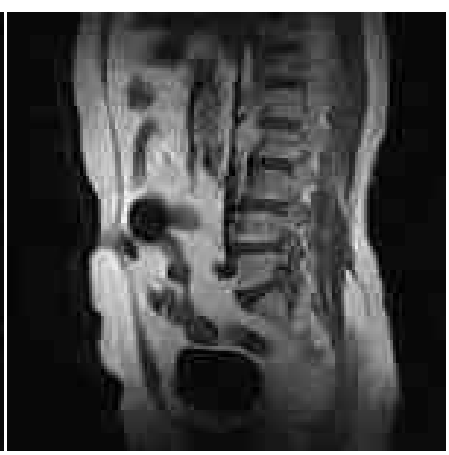

(d)

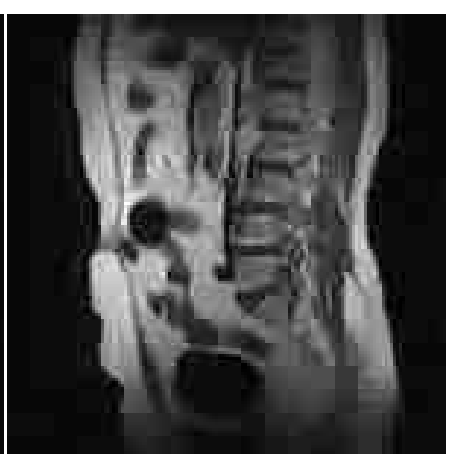

(f)
Figure2 (a) Original image1 (b) $\mathrm{CR}=1.48$ compressed image1 (c) $\mathrm{CR}=2.04$ compressed image 1 (d) $\mathrm{CR}=2.48$ compressed image 1 (e) $\mathrm{CR}=2.77$ compressed image1 (f) $\mathrm{CR}=3.01$ compressed image 1

been compressed using the algorithms combining CS \& DWT and CS \& DCT. Their performance is evaluated using PSNR, RMS and CoC parameters. The CS and DWT based compression algorithm outperforms the CS and DCT based algorithm as it achieved the highest compression ratio of 3.01 yielding the PSNR and $\mathrm{CoC}$ values 


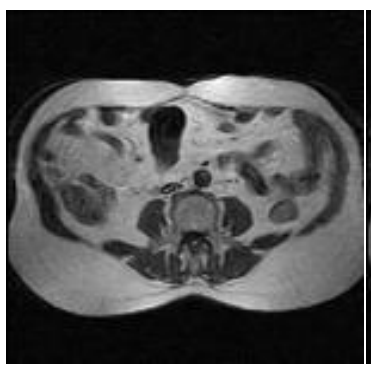

(a)

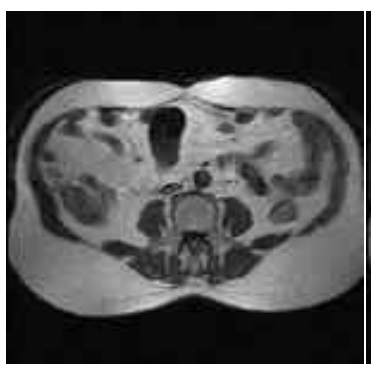

(c)

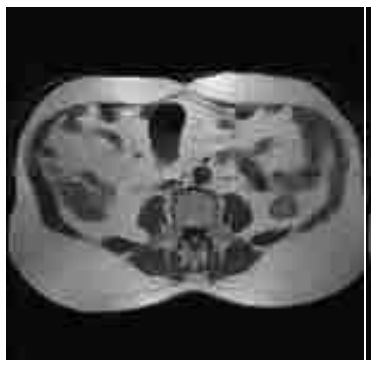

(e)

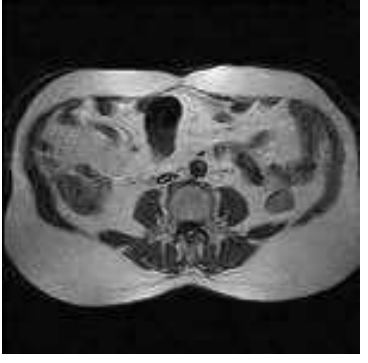

(b)

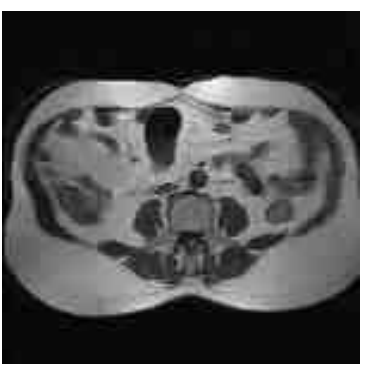

(d)

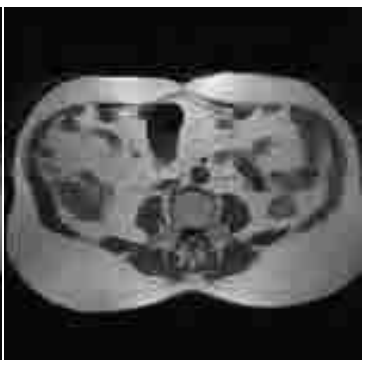

(f)
Figure 3 (a) Original image2 (b) $\mathrm{CR}=1.47$ compressed image2 (c) $\mathrm{CR}=1.99$ compressed image 2 (d) $\mathrm{CR}=2.42$ compressed image 2 (e) $\mathrm{CR}=2.68$ compressed image2 (f) $\mathrm{CR}=2.89$ compressed image 2

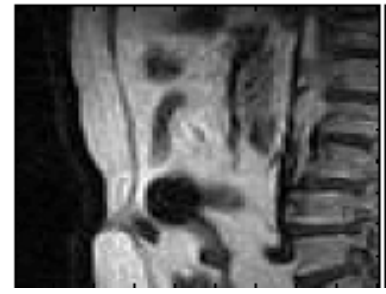

(a)

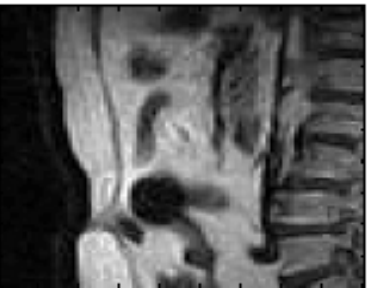

(b)
Figure4 (a) Original image1 (b) $\mathrm{CR}=1.5789$ compressed image1

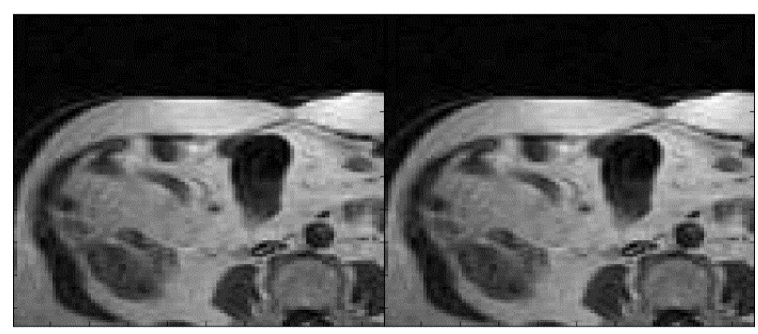

(a) (b)

Figure5 (a) Original image2 (b) $\mathrm{CR}=1.5789$ compressed image2

Table 4. Third level decomposition

\begin{tabular}{|c|c|c|c|}
\hline A3 & H3 & H2 & H1 \\
\hline V3 & D3 & D2 \\
\hline & V2 & D1 \\
\hline
\end{tabular}

Table 5. Performance of CS and DWT based compression algorithm

\begin{tabular}{|c|c|c|c|c|}
\hline & CR & PSNR, dB & RMS & CoC \\
\hline \multirow{4}{*}{ Image 1 } & 1.48 & 43.6326 & 1.6785 & 0.9998 \\
\cline { 2 - 5 } & 2.04 & 37.7404 & 3.3077 & 0.9992 \\
\cline { 2 - 5 } & 2.48 & 34.7934 & 4.6438 & 0.9984 \\
\cline { 2 - 5 } & 2.77 & 32.9042 & 5.7720 & 0.9975 \\
\hline \multirow{4}{*}{ Image2 } & 3.01 & 31.6732 & 6.6509 & 0.9966 \\
\cline { 2 - 5 } & 1.47 & 44.0629 & 1.5973 & 0.9999 \\
\cline { 2 - 5 } & 1.99 & 38.2155 & 3.1316 & 0.9994 \\
\cline { 2 - 5 } & 2.42 & 35.1415 & 4.4613 & 0.9989 \\
\cline { 2 - 5 } & 2.68 & 33.2121 & 5.5711 & 0.9982 \\
\hline
\end{tabular}

Table 6. Performance of CS and DWT based compression algorithm

\begin{tabular}{|c|c|c|c|c|}
\hline & CR & PSNR, dB & RMS & CoC \\
\hline Image 1 & 1.5789 & 13.6059 & 53.2407 & 0.8487 \\
\hline Image 2 & 1.5789 & 12.7490 & 58.7608 & 0.8280 \\
\hline
\end{tabular}

of 31.6732 and 0.9966 , respectively for an MRI image. For a CT image, this algorithm achieved a maximum PSNR and CoC values of 31.7935 and 0.9975 , respectively while compressing the image with $\mathrm{CR}=2.89$.

\section{References}

1. CandesE, Wakin M (2008)An introduction to Compressive Sampling.IEEE Signal Processing magazine25: 21-30.

2. Donoho D (2006) Compressed sensing.IEEE Transactions on Information theory 52 1289-1306.

3. Sapkal AM,Bairag VK (2009)Selection of Wavelets for medical image, Int. Conf. on Advances in Computing, Control and Telecommunication Technologies (ACT '09) Trivandrum India. 678-680.

4. http://www-inst.eecs.berkeley.edu/ ee225b / sp11/ lectures/ CSmeetsML-Lecture1/ codes/11magic/ 11magic.pdf

5. Huihui B, Anhong W, Mengmeng Z (2010)Compressive Sensing for DCT Image. Int Conf. on Computational Aspects of Social Networks (CASoN). 378-381.

6. Mayur MS, Falgun NT, Rahul KK, Chintan KM (2010)CT image compression using compressive sensing and wavelet transform. Int. Conf. on Communication Systems and Network Technologies (CSNT)138-142.

7. Rabiul SI, Huang X, Keng LO (2015)Image Compression Based on Compressive Sensing Using Wavelet Lifting Scheme.The International Journal of Multimedia \& its Applications 7: 1-16.

8. Qureshi MA, Deriche M (2016)A new wavelet based efficient image compression algorithm using compressive sensing.Multimedia Tools and Applications75: 6737 6754.

9. Deriche M, Qureshi MA, Azeddine B (2015)An image compression algorithm using reordered wavelet coefficients with compressive sensing.International Conference on Image Processing Theory, Tools and Applications (IPTA)498-503.

10. Shuling L, Baojun Z, Linbo T, Meiping J (2015) Adaptive image compression system based on TMS320C6713.IET International Radar Conference 1-5.

Copyright: (C2017 Kher R. This is an open-access article distributed under the terms of the Creative Commons Attribution License, which permits unrestricted use, distribution, and reproduction in any medium, provided the original author and source are credited. 